\title{
PET of EGFR Antibody Distribution in Head and Neck Squamous Cell Carcinoma Models
}

\author{
Gang Niu ${ }^{1}, Z i b o \mathrm{Li}^{2}$, Jin Xie ${ }^{1}$, Quynh-Thu Le ${ }^{3}$, and Xiaoyuan Chen ${ }^{1}$
}

${ }^{1}$ Molecular Imaging Program at Stanford (MIPS), Department of Radiology and Bio-X Program, Stanford University School of Medicine, Stanford, California; ${ }^{2}$ USC Molecular Imaging Center, Department of Radiology, Keck School of Medicine, Los Angeles, California; and ${ }^{3}$ Department of Radiation Oncology, Stanford University School of Medicine, Stanford, California

Epidermal growth factor receptor (EGFR) is a well-characterized protooncogene that has been shown to promote tumor progression in solid cancers. Clinical results for EGFR targeting with specific monoclonal antibodies (mAbs) such as cetuximab and panitumumab are promising; however, most studies indicate that only a subgroup of patients receiving the mAbs benefit from the immunotherapy, independent of EGFR expression level. To understand the in vivo kinetics of antibody delivery and localization, we performed small-animal PET studies with ${ }^{64} \mathrm{Cu}$-labeled panitumumab in xenografts derived from 3 cell lines of human head and neck squamous cell carcinoma (HNSCC). Methods: Nude mice bearing HNSCC tumors with different levels of EGFR expression were imaged with small-animal PET using ${ }^{64} \mathrm{Cu}-1,4,7,10$-tetraazacyclododecane$\mathrm{N}, \mathrm{N}^{\prime}, \mathrm{N}^{\prime \prime}, \mathrm{N}^{\prime \prime \prime}$-tetraacetic acid (DOTA)-panitumumab. Antibody distribution in the tumors was confirmed by ex vivo immunostaining using panitumumab and fluorescein 5(6)-isothiocyanate (FITC) panitumumab. CD31 immunostaining and Evans blue assay were also performed to assess the tumor vascular density and permeability. Results: Among these 3 tumor models, UM-SCC-22B tumors with the lowest EGFR protein expression showed the highest ${ }^{64} \mathrm{Cu}$-DOTA-panitumumab accumulation, whereas SQB20 tumors with the highest EGFR expression showed the lowest ${ }^{64} \mathrm{Cu}$-DOTA-panitumumab accumulation. Ex vivo staining demonstrated that SQB20 cells still had extremely high EGFR expression after forming tumors in nude mice, indicating that the low uptake of ${ }^{64} \mathrm{Cu}$-DOTA-panitumumab in SQB20 tumors was not due to the loss of EGFR expression. The results from CD31 immunostaining and Evans blue permeability assay suggest that the low vessel density, poor vascular permeability, and binding site barrier are likely responsible for the overall low tumor uptake of the highly EGFR-expressing SQB20 tumors. Conclusion: The results from this study provide a possible explanation for the lack of an observed correlation between therapeutic efficacy of cetuximab and panitumumab and EGFR expression level as determined by immunohistochemistry or fluorescent in situ hybridization and may shed new light on the complications of anti-EGFR mAb therapy for HNSCC and other malignancies.

Received Jan. 6, 2009; revision accepted Mar. 16, 2009.

For correspondence or reprints contact: Xiaoyuan Chen, Molecular Imaging Program at Stanford (MIPS), Department of Radiology and Bio-X Program, Stanford University School of Medicine, 1201 Welch Rd., P095,

Stanford, CA 94305-5484.

E-mail: shawchen@stanford.edu

COPYRIGHT () 2009 by the Society of Nuclear Medicine, Inc.
Key Words: epidermal growth factor receptor (EGFR); monoclonal antibody (mAb); positron emission tomography (PET); headneck squamous cell carcinoma (HNSCC); tumor binding barrier

J Nucl Med 2009; 50:1116-1123

DOI: 10.2967/jnumed.109.061820

$\mathbf{T}$ he epidermal growth factor receptor (EGFR) is a well-characterized protooncogene that has been shown to promote tumor progression in several solid cancers (1). EGFR is a member of the structurally related erbB family of receptor tyrosine kinases (2). It has been reported that more than $95 \%$ of head and neck squamous cell carcinomas (HNSCCs) express elevated EGFR levels, compared with the levels in normal mucosa (3). Further investigations show that the elevated EGFR expression is an independent indicator of poor prognosis and reduced survival in HNSCC patients (4). EGFR-targeted therapies include monoclonal antibodies (mAbs) such as cetuximab (IMC-C225; ImClone Systems Inc.) and panitumumab (ABX-EGF; Amgen Inc.), which block the extracellular ligand-binding domain of the receptor and tyrosine kinase inhibitors that prevent activation of the cytoplasmic kinase portion. These targeting approaches have shown great promise in preclinical studies $(5,6)$. In patients with locoregionally advanced HNSCC, the combination of cetuximab and high-dose radiation was found to yield survival superior to that of radiation alone (7). Similarly, the addition of cetuximab to chemotherapy resulted in significantly longer median survival when compared with chemotherapy alone in patients with recurrent or metastatic HNSCC in a large randomized study (8).

Even though clinical results for EGFR targeting with specific antibodies are promising, most studies indicate that only a subgroup of patients receiving the mAbs benefit from them $(9,10)$. To date, there is no known marker that can be used consistently to identify patients who would likely benefit from cetuximab therapy (11). No correlation has been found between the efficacy of cetuximab and EGFR tumoral staining intensity by immunohistochemistry $(11,12)$. In addition, a response to cetuximab has been observed in 
patients with EGFR-negative tumors (13). Although it has been reported that the EGFR gene copy number may predict the response to cetuximab, there are concerns about the reproducibility of such an assay (14). Most recently, KRAS mutations have been shown to be markers of resistance to anti-EGFR antibody in colorectal cancer; however, such a mutation is rare in HNSCC (15). Because EGFR antibody treatment is rather expensive and not without associated toxicity, a reliable marker would be quite helpful in the management of HNSCC patients.

It has been demonstrated that noninvasive molecular imaging can provide additional diagnostic information to improve patient management $(16,17)$. Such an imaging approach has also been applied to EGFR using SPECT with ${ }^{111} \mathrm{In}$ and ${ }^{99 \mathrm{~m} T c-l a b e l e d ~ E G F R-s p e c i f i c ~ a n t i b o d i e s ~(18-20) . ~}$ PET of EGFR has also been performed with cetuximab conjugated to a bifunctional chelator such as 1,4,7,10tetraazacyclododecane- $N, N^{\prime}, N^{\prime \prime}, N^{\prime \prime \prime}$-tetraacetic acid (DOTA) and labeled with ${ }^{64} \mathrm{Cu}(21,22)$. Small-animal PET showed that uptake of ${ }^{64} \mathrm{Cu}$-DOTA-cetuximab increased over time in EGFR-positive tumors but was relatively low in EGFRnegative tumors (23). ${ }^{64} \mathrm{Cu}$-DOTA-cetuximab has also been used to detect and quantify EGFR expression in cervical cancer tumors (21).

To fully understand the issue of antibody delivery in HNSCC and shed some light on the seemingly contradictory observation of EGFR expression and antibody therapeutic efficacy, we labeled panitumumab, a fully humanized $\mathrm{mAb}$ against EGFR (24), with ${ }^{64} \mathrm{Cu}$ and performed quantitative PET studies on different HNSCC tumor xenografts. We found that the in vivo uptake of labeled panitumumab in tumors failed to correlate with EGFR protein expression levels in HNSCC xenograft models.

\section{MATERIALS AND METHODS}

All commercially available chemical reagents were used without further purification. DOTA was purchased from Macrocyclics, Inc., and Chelex 100 resin (50-100 mesh) was purchased from Sigma-Aldrich. Water and all buffers were passed though a Chelex 100 column $(1 \times 15 \mathrm{~cm})$ before use in radiolabeling procedures to ensure that the aqueous buffer was free of heavy metals. PD-10 desalting columns were purchased from GE Healthcare. Athymic nude mice were obtained from Harlan at $4-6$ wk of age. ${ }^{64} \mathrm{Cu}$ was ordered from the University of Wisconsin-Madison. The human HNSCC cell lines SQB20, SAS, and UM-SCC-22B were obtained, respectively, from Dr. J. Martin Brown's laboratory at Stanford University; from the Cell Resource Center for the Biomedical Research Institute of Development, Aging, and Cancer, Tohoku University; and from the University of Michigan. The cell lines were maintained in DMEM medium supplemented with $10 \%$ fetal bovine serum, $1 \%$ glutamine, $100 \mathrm{U}$ of penicillin per milliliter, and $100 \mu \mathrm{g}$ of streptomycin per milliliter (Invitrogen).

\section{Antibody Labeling}

Fluorescein 5(6)-isothiocyanate (FITC), purchased from SigmaAldrich, was dissolved in anhydrous dimethyl sulfoxide immediately before use and then added to panitumumab with a ratio of 50 $\mu \mathrm{g}$ per mg of antibody. The mixture was incubated and rotated at room temperature for $60 \mathrm{~min}$ for covalent conjugation. The unreacted FITC was removed by PD-10 column. FITC-to-antibody ratio and antibody concentration were determined by the following equation after measuring the absorbance at 280 and $495 \mathrm{~nm}$ : antibody $(\mathrm{mg} / \mathrm{mL})=\left[\mathrm{A}_{280}-0.31 \times \mathrm{A}_{495}\right] / 1.4$; FITC-to-antibody ratio $=3.1 \times \mathrm{A}_{495} /\left(\mathrm{A}_{280}-0.31 \times \mathrm{A}_{495}\right)$. The FITC-to-antibody ratio is 10.13 for FITC-panitumumab.

Detailed procedures for DOTA conjugation and for measurement of DOTA number have been reported earlier $(23,25)$. The reaction ratio of DOTA to antibody is $200: 1 .{ }^{64} \mathrm{CuCl}_{2}(74 \mathrm{MBq})$ was diluted in $300 \mu \mathrm{L}$ of $0.1 \mathrm{M}$ sodium acetate buffer ( $\mathrm{pH} 6.5)$ and added to 50 $\mu \mathrm{g}$ of DOTA-panitumumab. The reaction mixture was incubated for $1 \mathrm{~h}$ at $40^{\circ} \mathrm{C}$ with constant shaking. ${ }^{64} \mathrm{Cu}$-DOTA-panitumumab was then purified by PD-10 column using phosphate-buffered saline (PBS) as the mobile phase. The labeling yield was calculated by dividing the decay-corrected radioactivity of ${ }^{64} \mathrm{Cu}$-DOTA-panitumumab by the total radioactivity used for reaction.

\section{Flow Cytometry}

HNSCC cells were harvested and washed with PBS containing $0.5 \%$ bovine serum albumin. On blockade by $2 \%$ bovine serum albumin in PBS, the cells were incubated with panitumumab (10 $\mu \mathrm{g} / \mathrm{mL}$ in PBS containing $2 \%$ bovine serum albumin). FITCconjugated donkey antihuman IgG (1:200) was then added and allowed to incubate for $1 \mathrm{~h}$ at room temperature. After washing, the cells were analyzed using an LSR flow cytometer (Beckman Coulter). The FITC signal intensity was analyzed using CellQuest software (version 3.3; Becton-Dickinson).

\section{Tumor Models}

All animal experiments were performed under a protocol approved by the Stanford University Administrative Panel on Laboratory Animal Care. Subcutaneous HNSCC tumor models were established in 4- to 6-wk-old female athymic nude mice. Typically, $5 \times 10^{6}$ cells suspended in $50 \mu \mathrm{L}$ of PBS were injected and the mice underwent PET when the tumor volume reached $200-400 \mathrm{~mm}^{3}$ (3-4 wk after inoculation).

\section{PET and Image Analysis}

PET of tumor-bearing mice was performed on a microPET R4 rodent model scanner (Siemens Medical Solutions) as described earlier (23). The mice were intravenously injected with ${ }^{64} \mathrm{Cu}-$ DOTA-panitumumab or ${ }^{64} \mathrm{Cu}-\mathrm{DOTA}-\mathrm{IgG}$ (Jackson ImmunoResearch Laboratories) $(7-8 \mathrm{MBq} / 6 \mu \mathrm{g} / \mathrm{mouse})$, and static scans were acquired at $4,20,30$, and $48 \mathrm{~h}$ after injection. For each scan, 3-dimensional regions of interest were drawn over the tumor, liver, heart, and muscle on decay-corrected whole-body coronal images. The average radioactivity concentration within a tumor or an organ was obtained from mean pixel values within the region-of-interest volume. These mean values were converted to counts $/ \mathrm{mL} / \mathrm{min}$ using a conversion factor. Assuming a tissue density of $1 \mathrm{~g} / \mathrm{mL}$, the counts $/ \mathrm{mL} / \mathrm{min}$ were converted to counts $/ \mathrm{g} / \mathrm{min}$ and then divided by the injected dose to obtain an imaging region-of-interestderived percentage injected dose (\%ID)/g.

\section{Measurement of Vascular Permeability Using Evans Blue}

The effective microvascular permeability of albumin-Evans blue was determined using the simplified indicator diffusion method (26). Evans blue (Sigma-Aldrich) was administered intravenously at a dose of $30 \mathrm{mg} / \mathrm{kg}$. The mice were reperfused at an elevated perfusion pressure with physiologic saline immediately before 
tumor excision to remove albumin-Evans blue from the tumor circulation. Tumors were excised $4 \mathrm{~h}$ after the dye had been injected and were cut into pieces weighing approximately $50 \mathrm{mg}$ to facilitate the extraction of Evans blue. The pieces were pooled in a tube and weighed before Evans blue was extracted in formamide $(0.01 \mathrm{~mL} / \mathrm{mg}$ of tumor tissue) for $72 \mathrm{~h}$. Relative Evans blue concentrations were determined by measuring the light absorbance at $620 \mathrm{~nm}$. For Evans blue fluorescence staining, tumors were harvested and sectioned at 4 $\mathrm{h}$ after dye injection. After being mounted with medium containing 4,6-diamino-2-phenylindole (DAPI), the sections were observed under an LSM 510 confocal microscope (Zeiss) with optical filters (543-nm excitation; long pass, 585-nm emission).

\section{Immunofluorescence Staining}

Frozen HNSCC tumor sections ( $5 \mu \mathrm{m}$ thick) were warmed to room temperature, fixed with ice-cold acetone for $10 \mathrm{~min}$, and dried in the air for $30 \mathrm{~min}$. The sections were rinsed in PBS for 2 min and blocked in $10 \%$ donkey serum for $1 \mathrm{~h}$ at room temperature. The sections were incubated with panitumumab $(10 \mu \mathrm{g} / \mathrm{mL})$ for $1 \mathrm{~h}$ at room temperature and visualized with FITC-conjugated donkey antihuman secondary antibody (1:200, Jackson ImmunoResearch Laboratories) under a microscope (Axiovert 200 M; Carl Zeiss USA). Images were acquired under the same conditions and displayed at the same scale.

For in vivo staining, $100 \mu \mathrm{g}$ of DOTA-panitumumab or FITCpanitumumab were injected by tail vein. The animals were sacrificed $30 \mathrm{~h}$ after injection, and the tumor samples were collected. The tumor sections with DOTA-panitumumab were fixed and stained using a similar procedure except that no primary antibody was added. The tumor sections with FITC-panitumumab were mounted with DAPI-containing mounting medium and were observed directly without any further staining.

\section{CD31 Staining and Microvascular Density Measurement}

Frozen slices ( $5 \mu \mathrm{m}$ thick) of HNSCC tumor were fixed with cold acetone for $10 \mathrm{~min}$ and dried in the air for $30 \mathrm{~min}$. The slices were rinsed with PBS for 2 min and blocked with 10\% donkey serum for $30 \mathrm{~min}$ at room temperature. The slices were then incubated with panitumumab and rat antimouse CD31 antibody for $1 \mathrm{~h}$ at room temperature and visualized using FITC-conjugated donkey antihuman secondary antibody (1:200; Jackson ImmunoResearch Laboratories, Inc.) and Cy3-conjugated rat antimouse IgG (1:200; Jackson ImmunoResearch Laboratories, Inc.).

After CD31 staining, 10 random views in both the center and the periphery of the tumor slices were selected for microvessel density (MVD) analysis using an observer-set threshold to distinguish vascular elements from surrounding tissue parenchyma. The vessel that contained branching points was counted as a single vessel. The number of vessels counted was divided by the field of view to yield the MVD, as vessels $/ \mathrm{mm}^{2}$.

\section{Statistical Analysis}

Quantitative data were expressed as mean \pm SD. Means were compared using 1-way ANOVA and the Student $t$ test. $P$ values less than 0.05 were considered statistically significant.

\section{RESULTS}

High Expression of EGFR in HNSCC Cell Lines

We selected 3 different HNSCC cell lines and analyzed their EGFR expression levels by FACS (Fig. 1). All 3 cell

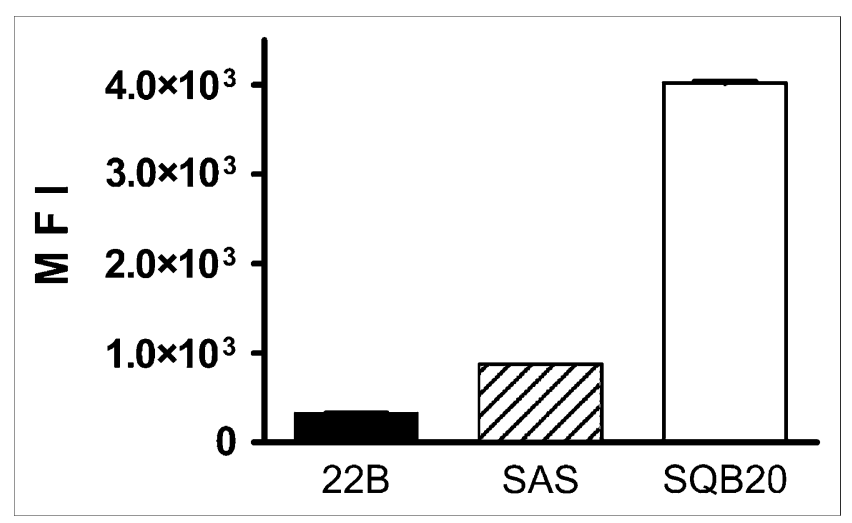

FIGURE 1. Flow cytometric analysis of EGFR expression on HNSCC cells. Panitumumab was used as primary antibody, and FITC-conjugated donkey antihuman IgG was used as secondary antibody. Mean values $( \pm \mathrm{SD}$ ) of FITC signal intensity (MFI) of 3 measurements are shown. 22B = UM-SCC-22B.

lines showed relatively high EGFR expression, in the order of SQB20 > SAS > UM-SCC-22B. Immunostaining of tumor sections derived from these cell lines also showed extremely high EGFR expression in SQB20 tumors, high expression in SAS tumors, and relatively low expression in UM-SCC-22B tumors.

\section{PET of EGFR Expression}

The specific activity of ${ }^{64} \mathrm{Cu}$-DOTA-panitumumab was $1.35 \pm 0.26 \mathrm{GBq} / \mathrm{mg}$, and the radiolabeling yield was $85.0 \% \pm 9.2 \%(n=5)$. The decay-corrected whole-body transaxial images containing the tumors are shown in Figure 2. At all time points, the accumulation of ${ }^{64} \mathrm{Cu}-$ DOTA-panitumumab was highest in UM-SCC-22B tumors, lowest in SQB20 tumors, and moderate in SAS tumors. Quantitative data based on region-of-interest analysis are shown in Table 1. At $30 \mathrm{~h}$ after injection, the UM-SCC-22B tumor uptake of ${ }^{64} \mathrm{Cu}$-DOTA-panitumumab was $31.42 \pm$ $10.77 \% \mathrm{ID} / \mathrm{g}$, SAS tumor uptake was $12.39 \pm 4.15 \% \mathrm{ID} / \mathrm{g}$, and SQB20 tumor uptake was $8.76 \pm 1.07 \% \mathrm{ID} / \mathrm{g}$. The liver also had prominent radioactivity accumulation, with an uptake of $11.96 \pm 3.87 \% \mathrm{ID} / \mathrm{g}$ at $30 \mathrm{~h}$ after injection, due to both the hepatic clearance of antibody-based tracer and possible transchelation. The blood activity concentration was $12.35 \pm 4.25 \% \mathrm{ID} / \mathrm{g}$ at $30 \mathrm{~h}$ after injection, indicating the long circulation life of the antibody.

We also imaged these HNSCC tumors with ${ }^{64} \mathrm{Cu}$-DOTAIgG to eliminate the influence of passive targeting for PET quantification. Compared with ${ }^{64} \mathrm{Cu}$-DOTA-panitumumab, ${ }^{64} \mathrm{Cu}$-DOTA-IgG had a similar blood concentration (13.01 \pm $1.28 \% \mathrm{ID} / \mathrm{g}$ at $30 \mathrm{~h})$ and liver uptake $(10.92 \pm 1.77 \% \mathrm{ID} / \mathrm{g}$ at $30 \mathrm{~h}$ ). The tumor uptake of ${ }^{64} \mathrm{Cu}-\mathrm{DOTA}-\mathrm{IgG}$ was low in all 3 tumor models. After IgG subtraction, UM-SCC-22B still showed the highest absolute uptake $(14.14 \pm 3.89 \% \mathrm{ID} / \mathrm{g})$, whereas SQB20 showed the lowest absolute uptake (2.85 \pm $0.82 \% \mathrm{ID} / \mathrm{g}$ ) (Fig. 2B). 
$4 \mathrm{~h}$
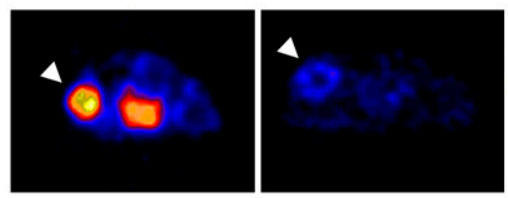

$20 \mathrm{~h}$
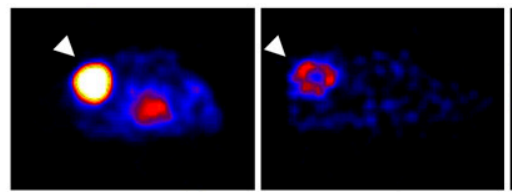

$30 \mathrm{~h}$
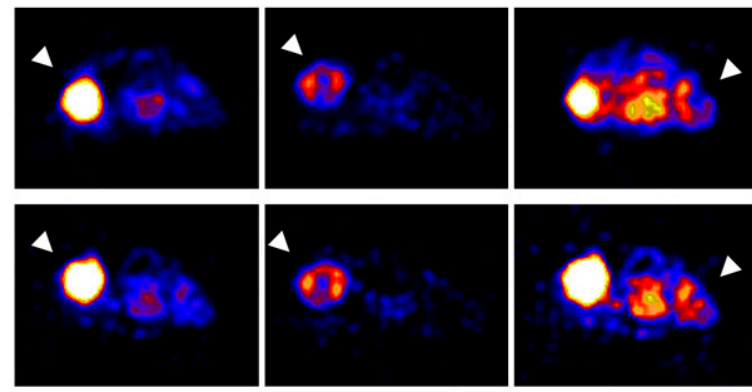

B

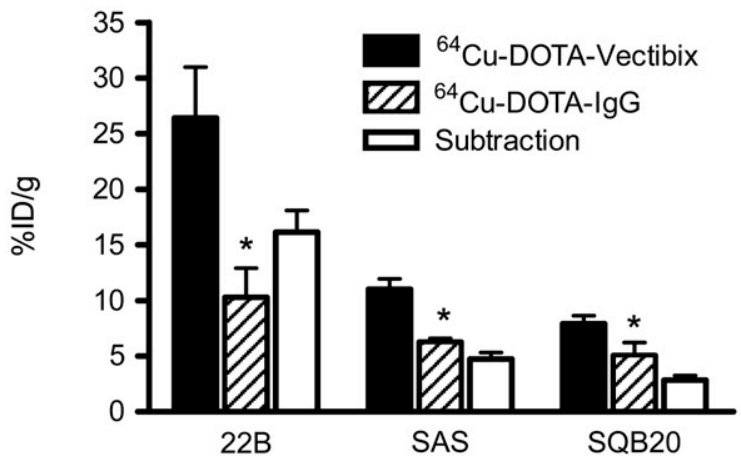

FIGURE 2. (A) Small-animal PET images of HNSCC tumorbearing nude mice at different time points after intravenous injection of ${ }^{64} \mathrm{Cu}$-DOTA-panitumumab ( $n=4 /$ group). Decaycorrected transaxial images at different time points are shown, and tumors are indicated by arrowheads. For UMSCC-22B and SAS tumors, scale ranged from $0 \% \mathrm{ID} / \mathrm{g}$ to $30 \% \mathrm{ID} / \mathrm{g}$, and for SQB20 tumors, scale ranged from $0 \% \mathrm{ID}$ to $15 \% \mathrm{ID} / \mathrm{g}$ for optimal visualization. (B) HNSCC tumor uptake levels of ${ }^{64} \mathrm{Cu}$-DOTA-panitumumab and ${ }^{64} \mathrm{Cu}-\mathrm{DOTA}-\mathrm{lgG}$ at $20 \mathrm{~h}$ after injection quantified from small-animal PET scans $(n=4) .22 \mathrm{~B}=$ UM-SCC-22B. ${ }^{*} P<0.05$.

\section{Panitumumab Distribution in HNSCC Tumors}

The PET data showed that uptake of ${ }^{64} \mathrm{Cu}$-DOTApanitumumab was highest in the UM-SCC-22B tumors that had the lowest EGFR protein expression. Inversely, the radioactivity accumulation was lowest in the SQB20 tumors that had the highest EGFR expression. To further explain this seemingly abnormal phenomenon, we conjugated panitumumab with a fluorescent dye FITC and harvested tumors at $30 \mathrm{~h}$ after intravenous injection of FITC-panitumumab. After being coated with a mounting medium containing DAPI, the tumor sections were examined under a fluorescence microscope and images were taken and displayed under the same conditions to make sure that the fluorescence intensity represented the FITCpanitumumab level. As shown in Figure 3B, for SQB20 tumors, FITC-panitumumab was primarily detected within several cell diameters of the blood vessels with high fluorescence density; for SAS tumors, the fluorescence signal was also limited to perivascular regions with a longer diffusive distance. In contrast, the fluorescence signal diffused much more homogeneously in UM-SCC22B tumors. DOTA-panitumumab showed an intratumoral distribution similar to that of FITC-panitumumab. Costaining with CD31 further demonstrated the limited perivascular localization of panitumumab in both SQB20 and SAS tumors (Fig. 3C and 3D). These results indicate that the poor penetration of the antibody through perivascular tissues is at least partially responsible for the low accumulation of ${ }^{64} \mathrm{Cu}$-DOTA-panitumumab in SQB20 tumors.

\section{Vascular Density and Permeability of HNSCC Tumors}

Vascular density is another determining factor for antibody diffusion and binding. The microvascular density (MVD) of HNSCC tumors was determined with CD31stained tumor sections. UM-SCC-22B tumors are highly vascularized, with an MVD of $27.1 / \mathrm{mm}^{2}$. SQB20 tumors have the lowest MVD, $4.7 / \mathrm{mm}^{2}$, and SAS tumors have an intermediate MVD of $12.5 / \mathrm{mm}^{2}$ (Fig. 4). The morphology of the vasculature also varied among these 3 tumor models.

Evans blue forms a complex with albumin in vivo by electrostatic interaction between the sulfonic acid group of the dye and the $\varepsilon$-amine groups of the lysine residues of albumin. The strong binding makes the behavior of Evans blue reflect the transport of albumin, which is about $67 \mathrm{kDa}$ with a diameter of about $7 \mathrm{~nm}$ (26). As shown in Figure 5A, the tumor vasculature was visualized through the highfluorescence signal of Evans blue, as is consistent with CD31 staining. In UM-SCC-22B tumors, moreover, the interstitial diffusion of Evans blue albumin from vessels can be identified. The quantification of Evan blue perfusion showed that UM-SCC-22B tumors were the most permeable among the 3 HNSCC tumor models, whereas SAS tumors were less permeable and SQB20 tumors were the least permeable (Fig. 5B).

\section{DISCUSSION}

In this study, we conjugated an EGFR-specific antibody, panitumumab, with DOTA and labeled the conjugate with ${ }^{64} \mathrm{Cu}$. Small-animal PET was performed to evaluate EGFR expression in tumor models derived from 3 HNSCC cell lines with different levels of EGFR expression. We found no correlation between PET quantification and EGFR protein expression level. To our surprise, UM-SCC-22B tumors, which had the lowest EGFR protein expression, showed the highest accumulation of ${ }^{64} \mathrm{Cu}$-DOTA-panitumumab accumulation whereas SQB20 tumors, with the highest EGFR expression, showed the lowest accumulation of ${ }^{64} \mathrm{Cu}$-DOTApanitumumab. The low uptake of ${ }^{64} \mathrm{Cu}$-DOTA-panitumumab 


\begin{tabular}{|c|c|c|c|c|c|c|c|c|}
\hline \multirow[b]{2}{*}{ Site } & \multicolumn{4}{|c|}{${ }^{64} \mathrm{Cu}$-DOTA-panitumumab (\%ID/g) } & \multicolumn{4}{|c|}{${ }^{64} \mathrm{Cu}-\mathrm{DOTA}-\lg \mathrm{g}(\% \mathrm{ID} / \mathrm{g})$} \\
\hline & $4 \mathrm{~h}$ & $20 \mathrm{~h}$ & $30 \mathrm{~h}$ & $48 \mathrm{~h}$ & $4 \mathrm{~h}$ & $20 \mathrm{~h}$ & $30 \mathrm{~h}$ & $48 \mathrm{~h}$ \\
\hline Blood & $22.73 \pm 3.99$ & $14.42 \pm 4.47$ & $12.35 \pm 4.25$ & $10.29 \pm 4.04$ & $20.54 \pm 2.58$ & $13.84 \pm 2.24$ & $13.01 \pm 1.28$ & $11.49 \pm 1.99$ \\
\hline Liver & $15.94 \pm 3.35$ & $12.52 \pm 3.29$ & $11.96 \pm 3.87$ & $11.55 \pm 3.64$ & $13.77 \pm 2.04$ & $10.42 \pm 1.17$ & $10.92 \pm 1.77$ & $10.83 \pm 2.25$ \\
\hline Muscle & $3.32 \pm 1.50$ & $2.479 \pm 1.08$ & $2.79 \pm 1.31$ & $2.14 \pm 1.03$ & $2.63 \pm 0.78$ & $2.20 \pm 0.40$ & $2.72 \pm 0.83$ & $2.10 \pm 0.11$ \\
\hline 22B & $16.09 \pm 6.52$ & $26.41 \pm 9.16$ & $31.42 \pm 10.77$ & $34.80 \pm 9.26$ & $5.70 \pm 3.92$ & $10.27 \pm 5.27$ & $11.75 \pm 6.35$ & $12.14 \pm 6.71$ \\
\hline SAS & $6.39 \pm 1.60^{*}$ & $11.01 \pm 1.84^{*}$ & $12.39 \pm 4.15^{\star}$ & $15.35 \pm 3.33^{*}$ & $3.51 \pm 0.86$ & $6.26 \pm 0.68$ & $7.20 \pm 2.25$ & $8.05 \pm 3.75$ \\
\hline SQB20 & $4.02 \pm 1.87^{\dagger}$ & $7.92 \pm 1.48^{\dagger}$ & $8.76 \pm 1.07^{\dagger}$ & $9.39 \pm 1.44^{\dagger}$ & $3.54 \pm 1.04$ & $5.07 \pm 2.30$ & $7.26 \pm 5.15$ & $8.57 \pm 5.04$ \\
\hline $\begin{array}{l}{ }^{*} P<0 \\
{ }^{\dagger} P<0 \\
\text { 22B }= \\
\text { Data a }\end{array}$ & $\begin{array}{l}05 . \\
.01 . \\
\text { UM-SCC-22B. } \\
\text { re \%ID/g and re }\end{array}$ & resent mean + & SD of 4 mice. & & & & & \\
\hline
\end{tabular}

in SQB20 tumors was not due to the loss of EGFR expression. On the contrary, ex vivo staining using panitumumab as the primary antibody demonstrated that SQB20 cells still had extremely high EGFR expression after forming tumors in nude mice. These results indicated that the expression level of EGFR cellular protein correlated poorly with the efficiency of antibody delivery to the tumors. Similar findings have also been reported for ${ }^{111}$ In-conjugated anti-EGFR antibody in breast cancer models (20).
At present, antibodies are indisputably the best-established class of binding molecules for tumor diagnosis and therapy $(27,28)$. Tumor antigen density is known to influence antibody-based tumor targeting (29). The expression level of EGFR in all 3 HNSCC cell lines tested was quite high compared with the other solid tumors. We have previously performed PET with ${ }^{64} \mathrm{Cu}$-DOTA-cetuximab on another 7 different solid tumor models, including U87MG human glioblastoma; PC-3 human prostate carci-

FIGURE 3. Immunofluorescence examination of EGFR expression and panitumumab localization in HNSCC tumors. Images were obtained under same conditions and displayed at same magnification and scale (except D). (A) Tumor sections were directly stained with panitumumab as primary antibody and with FITC-conjugated donkey antihuman $\operatorname{lgG}$ as secondary antibody. Murine CD31 was stained with Cy3-conjugated IgG to visualize tumor vasculature. SQB20 tumors showed highest fluorescence intensity, corresponding to highest EGFR expression. (B) Thirty hours after FITCpanitumumab injection, tumors were harvested and tumor sections were observed after being mounted with DAPI-containing medium. (C) Thirty hours after DOTApanitumumab injection, tumors were harvested and tumor sections were stained with FITC-conjugated donkey antihuman IgG. (D) In high-magnification view of images shown in $\mathrm{C}$, color was rescaled to emphasize relationship of panitumumab and vasculature (red from Cy3 for CD31; green from FITC for EGFR and panitumumab; blue from DAPI for nucleus visualization).
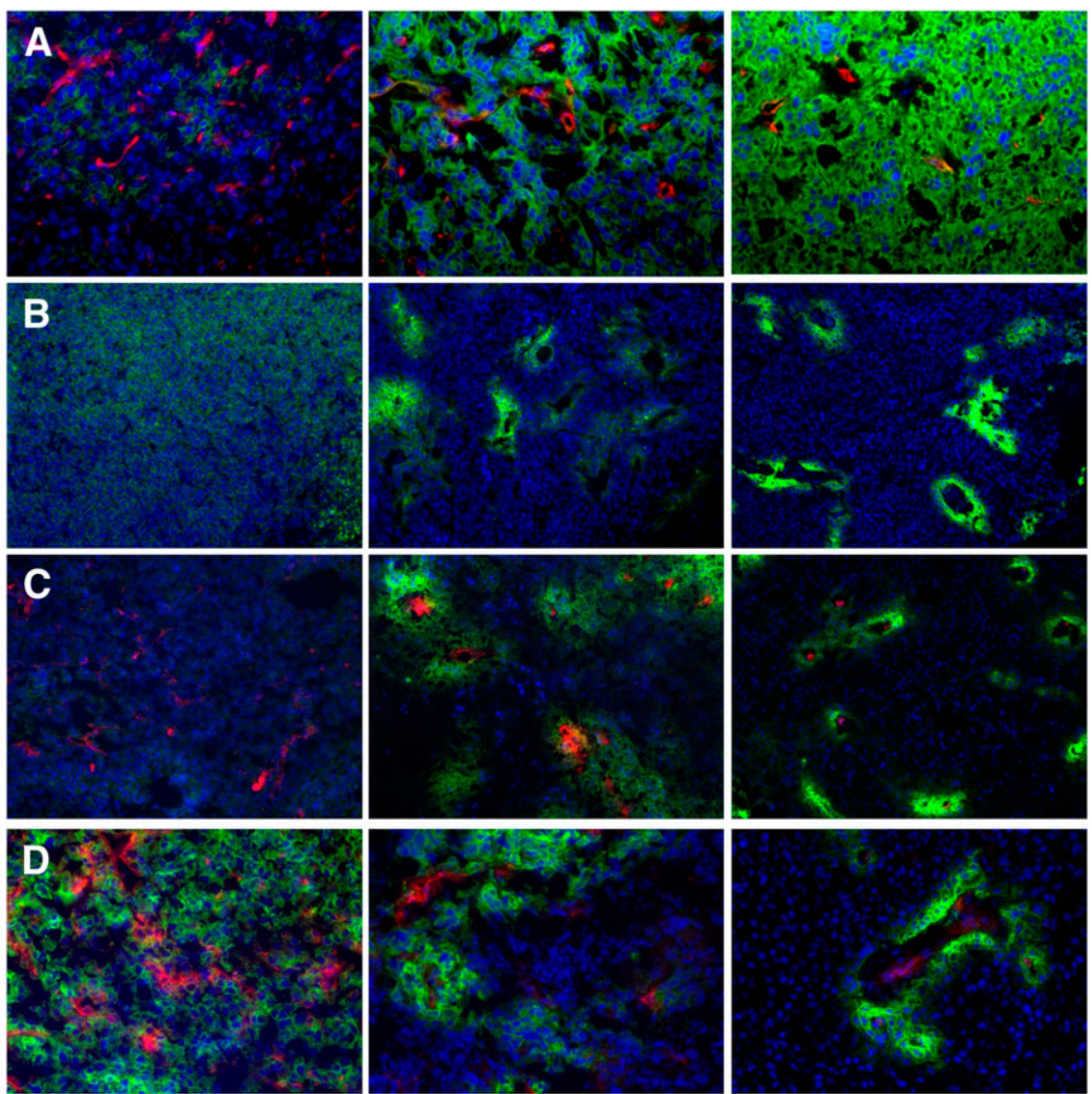

UM-SCC-22B
SAS

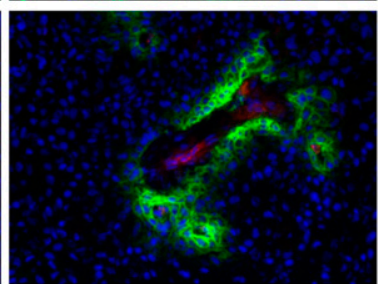

SQB20 


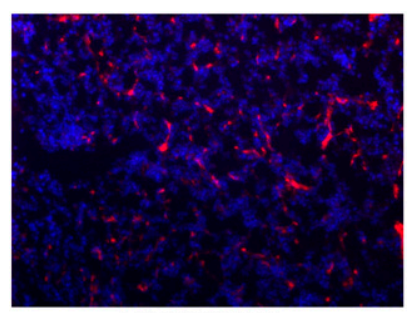

UM-SCC-22B

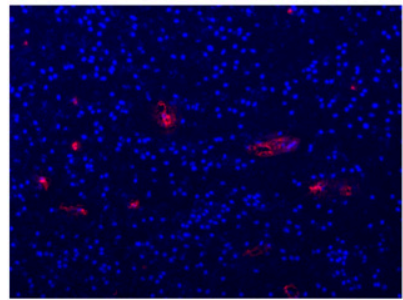

SQB20

FIGURE 4. MVD measurement of HNSCC tumors. Frozen slices of HNSCC tumor were stained with rat antimouse CD31 antibody and visualized using Cy3-conjugated rat antimouse IgG. After CD31 staining, 10 random views in both center and periphery of tumor slices were selected for MVD analysis using observer-set threshold to distinguish vascular elements from surrounding tissue parenchyma. Number of vessels counted was divided by field of view to yield MVD, as number of vessels $/ \mathrm{mm}^{2}$. 22B = UM-SCC22B. ${ }^{*} P<0.05$. ${ }^{\star} P<0.05$. ${ }^{* *} P<0.01$.

noma; CT-26 murine colorectal carcinoma; HCT-8, HCT-116, and SW620 human colorectal carcinoma; and MDA-MB435 human breast cancer (23). We found an increased accumulation of activity in EGFR-positive tumors over time but relatively low uptake in EGFR-negative tumors at all time points examined. Moreover, tracer uptake as measured by PET showed a good linear correlation with EGFR protein expression level as measured by Western blotting. Eiblmaier et al. (21) have also shown that EGFR messenger RNA expression corresponded directly to EGFR densities and levels of internalization of ${ }^{64} \mathrm{Cu}$-DOTAcentuximab in 5 cervical cancer cell lines. When compared between the 3 HNSCC cell lines and these other cell lines, the levels of EGFR expression were similar between the lowest-expressing HNSCC cells (UM-SCC22B) and the highest-expressing non-HNSCC cells (PC-3 prostate cancer). We speculated that antigen density is a major determining factor in PET with radionuclide-labeled antibodies when antigen expression level is below a certain threshold. Such a threshold may have been exceeded in the HNSCCs tested here. With regard to antibody binding affinity, we have tested ${ }^{64} \mathrm{Cu}$-DOTA-cetuximab in these 3 HNSCC models and the results were similar to those obtained with ${ }^{64} \mathrm{Cu}$-DOTApanitumumab, despite the fact that panitumumab has a higher affinity for EGFR than does cetuximab. Again, if antigen density exceeds a certain threshold, binding affinity may play a smaller role in antibody distribution.

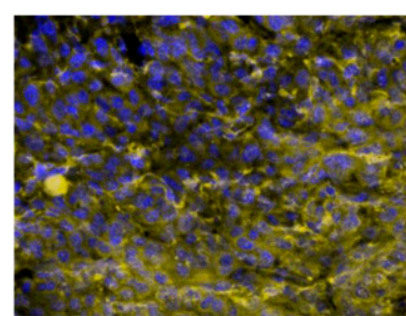

UM-SCC-22B

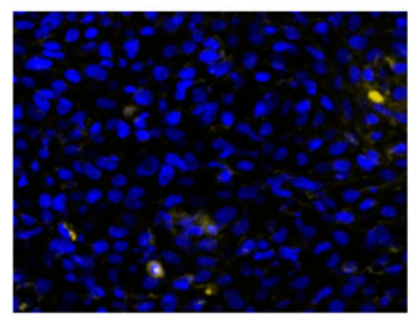

SQB20

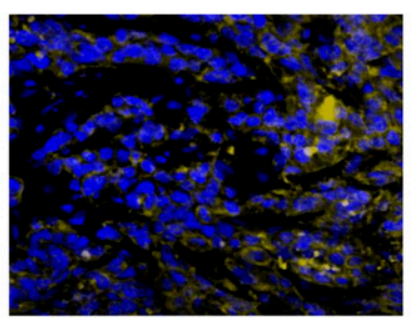

SAS

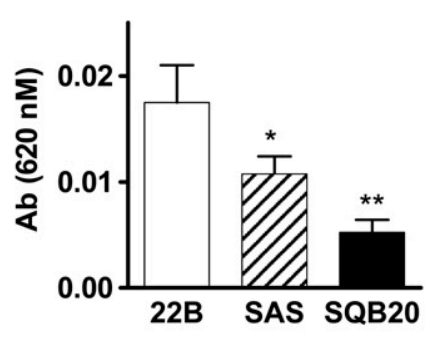

FIGURE 5. Measurement of vascular permeability using Evans blue. Tumors were excised $4 \mathrm{~h}$ after dye had been injected. After being mounted with medium containing DAPI, sections were observed under LSM 510 (Zeiss) confocal microscope with optical filters (543-nm excitation; long pass, $585-\mathrm{nm}$ emission). For quantification, Evans blue was extracted in formamide $(0.01 \mathrm{~mL} / \mathrm{mg}$ of tumor tissue) for 72 h. Relative Evans blue concentrations were determined by measuring light absorbance at $620 \mathrm{~nm}$. 22B = UM-SCC-22B. ${ }^{*} P<0.05$. ${ }^{\star *} P<0.01$.

Because of the lack of correlation between antigen density, binding affinity, and imaged antibody distribution, we surmised that low MVD and poor vascular permeability were mainly responsible for the observed imaging results. Compared with the other 2 HNSCC models, SQB20 tumors were poorly vascularized as indicated by CD31 staining. In addition, the vascular permeability of SQB20 tumors was the worst among the 3 tumor models, on the basis of Evans blue perfusion data. Apart from MVD and vascular permeability, we also speculated that there exists a binding-site barrier for ${ }^{64} \mathrm{Cu}$-DOTApanitumumab penetration in SQB20 tumors. The highaffinity binding of mAbs to the first encountered tumor antigen would retard antibody percolation (30), resulting in a more heterogeneous distribution. In the case of SQB20 tumors, the high number of EGFR receptors on the tumor cell surface may act as a barrier against distal diffusion of antibody. As shown in Figure 2, most panitumumab molecules were perivascular and diffused through a distance of only several cells. The implication is that the perivascular tumor cells depleted mAbs quickly and inhibited further penetration of panitumumab. The localization of panitumumab in SQB20 and UM-SCC-22B tumors is illustrated in Supplemental Figure 1 (supplemental materials are available online only at http://jnm.snmjournals.org). Because of the abundant blood supply, high MVD, and high blood vessel permeability, UMSCC-22B tumors showed a noticeably higher accumulation of ${ }^{64} \mathrm{Cu}$-DOTA-panitumumab than did the other $2 \mathrm{HNSCC}$ tumor 
models. Compared with a therapeutic dose of antibody (100$200 \mu \mathrm{g}$ per mouse), the imaging dose was very low ( $\sim 5 \mu \mathrm{g}$ per mouse). The difference in antibody concentration should be considered when one is integrating imaging data into therapeutic scenarios, because images with low concentrations of antibody may exaggerate the influence of vascular density and permeability.

Under physiologic conditions, EGFR is internalized on ligand activation and ligand-receptor complexes are targeted for lysosomal degradation, resulting in signal attenuation (31). A similar phenomenon has been observed for EGFR binding antibody (32). ${ }^{64} \mathrm{Cu}$-DOTA-cetuximab internalization was reported in cervical cancer cells and correlated well with EGFR expression, with approximately $25 \%$ of cell-surface EGFR being internalized after $4 \mathrm{~h}$ of incubation in all cell lines tested (21). We also evaluated panitumumab internalization in HNSCC cell lines and found that all HNSCC cells showed a similar internalization ratio of around $30 \%$ after $2 \mathrm{~h}$ of incubation at $37^{\circ} \mathrm{C}$ (Supplemental Fig. 2). Therefore, antibody internalization after panitumumab binding to EGFR was unlikely to have contributed to the differential distribution of ${ }^{64} \mathrm{Cu}$-DOTApanitumumab in HNSCC tumor models (33).

PET with radiolabeled antibody visualizes and quantifies several parameters, including tumor-specific binding, perfusion, vascularity, vascular permeability, and plasma halflife. Moreover, radiolabeled antibody PET provides more comprehensive information for patient screening and therapeutic guidance than does immunohistochemistry or fluorescent in situ hybridization - techniques that only sample antigen density in a small part of the tumor. The results from this study may provide a possible explanation for the lack of an observed correlation between therapeutic efficacy of cetuximab and panitumumab and EGFR expression level as determined by immunohistochemistry or fluorescent in situ hybridization (34). For tumors such as SQB20, even with high EGFR expression, the patchy and incomplete tumor perfusion could result in suboptimal therapeutic effects when therapeutic efficacy is dependent on uniform delivery to tumor cells $(30,35)$. We are pursuing studies to determine whether the results of ${ }^{64} \mathrm{Cu}$-DOTA-panitumumab or ${ }^{64} \mathrm{Cu}$-DOTA-cetuximab imaging correlate with treatment efficacy when the same antibody is used alone or conjugated to a high-energy radioisotope for radioimmunotherapy.

\section{CONCLUSION}

The in vivo tumor uptake quantified from PET with ${ }^{64} \mathrm{Cu}-$ DOTA-panitumumab in HNSCC tumor models failed to reflect the expression level of EGFR in vivo. The results revealed a possible explanation for the lack of an observed correlation between therapeutic efficacy of cetuximab and panitumumab and EGFR expression level as determined by immunohistochemistry or fluorescent in situ hybridization. This study may shed new light on the complications of antiEGFR mAb therapy for HNSCC and other malignancies.

\section{ACKNOWLEDGMENTS}

This project was supported in part by the National Cancer Institute (R21 CA102123, P50 CA114747, U54 CA119367, R24 CA93862, and R01 CA118582) and by a Department of Defense Prostate Cancer Postdoctoral Training Award.

\section{REFERENCES}

1. Salomon DS, Brandt R, Ciardiello F, Normanno N. Epidermal growth factorrelated peptides and their receptors in human malignancies. Crit Rev Oncol Hematol. 1995;19:183-232.

2. Matheny KE, Barbieri CE, Sniezek JC, Arteaga CL, Pietenpol JA. Inhibition of epidermal growth factor receptor signaling decreases p63 expression in head and neck squamous carcinoma cells. Laryngoscope. 2003;113:936-939.

3. Grandis JR, Tweardy DJ. Elevated levels of transforming growth factor alpha and epidermal growth factor receptor messenger RNA are early markers of carcinogenesis in head and neck cancer. Cancer Res. 1993;53:3579-3584.

4. Grandis JR, Zeng Q, Drenning SD, Tweardy DJ. Normalization of EGFR mRNA levels following restoration of wild-type p53 in a head and neck squamous cell carcinoma cell line. Int J Oncol. 1998;13:375-378.

5. Heimberger AB, Learn CA, Archer GE, et al. Brain tumors in mice are susceptible to blockade of epidermal growth factor receptor (EGFR) with the oral, specific, EGFR-tyrosine kinase inhibitor ZD1839 (Iressa). Clin Cancer Res. 2002;8:3496-3502.

6. Luwor RB, Johns TG, Murone C, et al. Monoclonal antibody 806 inhibits the growth of tumor xenografts expressing either the de2-7 or amplified epidermal growth factor receptor (EGFR) but not wild-type EGFR. Cancer Res. 2001;61:5355-5361.

7. Bonner JA, Harari PM, Giralt J, et al. Radiotherapy plus cetuximab for squamouscell carcinoma of the head and neck. N Engl J Med. 2006;354:567-578.

8. Vermorken JB, Mesia R, Rivera F, et al. Platinum-based chemotherapy plus cetuximab in head and neck cancer. N Engl J Med. 2008;359:1116-1127.

9. Folprecht G, Lutz MP, Schoffski $P$, et al. Cetuximab and irinotecan/5fluorouracil/folinic acid is a safe combination for the first-line treatment of patients with epidermal growth factor receptor expressing metastatic colorectal carcinoma. Ann Oncol. 2006;17:450-456.

10. Cohenuram M, Saif MW. Panitumumab the first fully human monoclonal antibody: from the bench to the clinic. Anticancer Drugs. 2007;18:7-15.

11. Saltz LB, Meropol NJ, Loehrer PJ Sr, Needle MN, Kopit J, Mayer RJ. Phase II trial of cetuximab in patients with refractory colorectal cancer that expresses the epidermal growth factor receptor. J Clin Oncol. 2004;22:1201-1208.

12. Cunningham D, Humblet Y, Siena S, et al. Cetuximab monotherapy and cetuximab plus irinotecan in irinotecan-refractory metastatic colorectal cancer. N Engl J Med. 2004;351:337-345.

13. Chung KY, Shia J, Kemeny NE, et al. Cetuximab shows activity in colorectal cancer patients with tumors that do not express the epidermal growth factor receptor by immunohistochemistry. J Clin Oncol. 2005;23:1803-1810.

14. Moroni M, Veronese S, Benvenuti S, et al. Gene copy number for epidermal growth factor receptor (EGFR) and clinical response to antiEGFR treatment in colorectal cancer: a cohort study. Lancet Oncol. 2005;6:279-286.

15. Lievre A, Bachet JB, Boige V, et al. KRAS mutations as an independent prognostic factor in patients with advanced colorectal cancer treated with cetuximab. J Clin Oncol. 2008;26:374-379.

16. Adams GP, Schier R, McCall AM, et al. High affinity restricts the localization and tumor penetration of single-chain fv antibody molecules. Cancer Res. 2001;61:4750-4755.

17. Erjala K, Sundvall M, Junttila TT, et al. Signaling via ErbB2 and ErbB3 associates with resistance and epidermal growth factor receptor (EGFR) amplification with sensitivity to EGFR inhibitor gefitinib in head and neck squamous cell carcinoma cells. Clin Cancer Res. 2006;12:4103-4111.

18. Goldenberg A, Masui H, Divgi C, Kamrath H, Pentlow K, Mendelsohn J. Imaging of human tumor xenografts with an indium-111-labeled anti-epidermal growth factor receptor monoclonal antibody. J Natl Cancer Inst. 1989;81:1616-1625.

19. Scopinaro F, De Vincentis G, Banci M, et al. In vivo study of a technetium labelled anti-EGFr MoAB. Anticancer Res. 1997;17:1761-1765.

20. Reilly RM, Kiarash R, Sandhu J, et al. A comparison of EGF and MAb 528 labeled with ${ }^{111}$ In for imaging human breast cancer. J Nucl Med. 2000;41:903-911.

21. Eiblmaier M, Meyer LA, Watson MA, Fracasso PM, Pike LJ, Anderson CJ. Correlating EGFR expression with receptor-binding properties and internalization of ${ }^{64} \mathrm{Cu}$-DOTA-cetuximab in 5 cervical cancer cell lines. J Nucl Med. 2008;49:1472-1479.

22. Ping Li W, Meyer LA, Capretto DA, Sherman CD, Anderson CJ. Receptorbinding, biodistribution, and metabolism studies of ${ }^{64} \mathrm{Cu}$-DOTA-cetuximab, a 
PET-imaging agent for epidermal growth-factor receptor-positive tumors. Cancer Biother Radiopharm. 2008;23:158-171.

23. Cai W, Chen K, He L, Cao Q, Koong A, Chen X. Quantitative PET of EGFR expression in xenograft-bearing mice using ${ }^{64} \mathrm{Cu}$-labeled cetuximab, a chimeric anti-EGFR monoclonal antibody. Eur J Nucl Med Mol Imaging. 2007;34:850858.

24. Yang XD, Jia XC, Corvalan JR, Wang P, Davis CG. Development of ABX-EGF, a fully human anti-EGF receptor monoclonal antibody, for cancer therapy. Crit Rev Oncol Hematol. 2001;38:17-23.

25. Cai W, Wu Y, Chen $\mathrm{K}$, Cao Q, Tice DA, Chen $\mathrm{X}$. In vitro and in vivo characterization of ${ }^{64} \mathrm{Cu}$-labeled Abegrin, a humanized monoclonal antibody against integrin alpha v beta 3. Cancer Res. 2006;66:9673-9681.

26. Graff BA, Bjornaes I, Rofstad EK. Microvascular permeability of human melanoma xenografts to macromolecules: relationships to tumor volumetric growth rate, tumor angiogenesis, and VEGF expression. Microvasc Res. 2001;61:187-198.

27. Wu AM, Olafsen T. Antibodies for molecular imaging of cancer. Cancer J. 2008;14:191-197.

28. Bernier J. Drug insight: cetuximab in the treatment of recurrent and metastatic squamous cell carcinoma of the head and neck. Nat Clin Pract Oncol. 2008;5:705-713.
29. Jain RK. Physiological barriers to delivery of monoclonal antibodies and other macromolecules in tumors. Cancer Res. 1990;50(3, suppl)814s-819s.

30. Fujimori K, Covell DG, Fletcher JE, Weinstein JN. A modeling analysis of monoclonal antibody percolation through tumors: a binding-site barrier. $\mathrm{J} \mathrm{Nucl}$ Med. 1990;31:1191-1198.

31. Stoscheck CM, Carpenter G. Down regulation of epidermal growth factor receptors: direct demonstration of receptor degradation in human fibroblasts. J Cell Biol. 1984;98:1048-1053.

32. Lammerts van Bueren JJ, Bleeker WK, Bogh HO, et al. Effect of target dynamics on pharmacokinetics of a novel therapeutic antibody against the epidermal growth factor receptor: implications for the mechanisms of action. Cancer Res. 2006;66:7630-7638.

33. Wheeler DL, Huang S, Kruser TJ, et al. Mechanisms of acquired resistance to cetuximab: role of HER (ErbB) family members. Oncogene. 2008;27:3944-3956.

34. Chung $\mathrm{CH}$, Ely $\mathrm{K}$, McGavran L, et al. Increased epidermal growth factor receptor gene copy number is associated with poor prognosis in head and neck squamous cell carcinomas. J Clin Oncol. 2006;24:4170-4176.

35. Juweid M, Neumann R, Paik C, et al. Micropharmacology of monoclonal antibodies in solid tumors: direct experimental evidence for a binding site barrier. Cancer Res. 1992;52:5144-5153. 